\title{
A Health Equity Primer: Understanding and Addressing Inequities in Pediatric Orthopaedics
}

\author{
Rachel L. Montoya, MSc ${ }^{1,2}$; Laura Gill-Sealy, $\mathrm{MD}^{3}$; Coleen S. Sabatini, MD, MPH',4 \\ ${ }^{1}$ Warren Alpert Medical School of Brown University, Providence, Rl; ${ }^{2}$ UCSF Center for Health Equity in Surgery and \\ Anesthesia (CHESA), San Francisco, CA; ${ }^{3}$ Pediatric Specialists of Virginia, Fairfax, VA; ${ }^{4}$ University of California San Francisco, \\ Department of Orthopaedic Surgery, UCSF Benioff Children's Hospital Oakland, Oakland, CA \\ Correspondence to: Coleen S. Sabatini, MD, MPH; 747 52nd Street, OPC 1st Floor, Oakland, CA 94609 U.S, \\ E-mail: Coleen.Sabatini@ucsf.edu
}

Received: January 17, 2022; Accepted: January 17, 2022; Published: February 1, 2022

DOI: 10.55275/JPOSNA-2022-0014

\begin{abstract}
:
Sociopolitical events in recent years including ongoing killings of Black Americans, violence against immigrants, and the COVID-19 pandemic have all shone a spotlight on the reality that we live in a society where an individual's safety, health, and ability to access and receive quality medical care are contingent upon their socioeconomic circumstances and personal identities. These events have inspired both increased civic engagement addressing structural inequities in healthcare, as well as changes within modalities of care delivery, making this an opportune time to examine health inequities in pediatric orthopaedics and re-commit to working towards their reduction. In order to ensure that orthopaedic surgeons are best positioned to engage in such work, this paper will discuss health equity in pediatric orthopaedics to serve as a foundational primer to inform future equity-focused efforts.

We will define health equity, equality, and intersectionality, and clarify the differences between these terms and their roles as lenses to better understand patients' unique backgrounds and personal circumstances. Existing research on health disparities in orthopaedics will be reviewed with a focus on the ways in which race, ethnicity, insurance status, gender and sexuality, health literacy, language, nutrition and food availability, physical ability, and environmental and personal safety all impact the accessibility and quality of orthopaedic care. Beyond discussing existing disparities, the paper will detail tangible actions that clinicians can take to improve inclusivity and accessibility in clinical environments and patient encounters. By centering an intersectional approach to patient care and integrating equity-focused changes within clinical settings, surgeons can ultimately improve their interpersonal interactions with marginalized patients and increase both the quality of the care that they provide and patient trust in that care.
\end{abstract}




\section{Key Concepts:}

- Engaging in health equity related work in pediatric orthopaedics requires an understanding of the differences between equity and equality and an intersectional approach to understanding patient identities and circumstances.

- Existing research within orthopaedics has demonstrated disparities in care access and surgical outcomes dependent on a patient's race, language, insurance status, and level of health literacy.

- Beyond just demonstrating the existence of health inequities, future research within orthopaedics should assess the efficacy and impact of tangible interventions aimed at reducing health disparities within orthopaedic settings.

- Orthopaedic surgeons should adopt a multifaceted approach to inequity-reduction that centers principles of equity and intersectionality interpersonally in patient interactions, logistically at the level of clinic scheduling and accessibility, and macroscopically through advocacy work at the local and national levels.

Physicians are bestowed with the privilege and responsibility of their patients' trust and well-being. To honor this trust, it is imperative that we strive to provide the highest quality and most equitable care possible. For orthopaedic surgeons, this goal requires a foundational understanding of health equity, and inequities in musculoskeletal health specifically, as well as an ongoing commitment to engaging in actionable work in clinical practice aimed at reducing those inequities. Past equity-focused research and advocacy in orthopaedics has often focused on increasing diversity among practicing surgeons, while patient-focused research has tended to be limited in scope, examining disparities in patient outcomes following a single procedure or access variability based on a single identity or demographic. ${ }^{1-7}$ This paper will seek to take a broader lens to the issue of health equity in pediatric orthopaedics in the United States and serve as an educational primer for practitioners that facilitates a comprehensive and informed approach to engaging in health-equity focused work moving forward.

This current historical juncture represents an apt time to examine these issues given the context of the COVID-19 pandemic and resultant changes in healthcare access and delivery, with exacerbation of numerous social and economic inequalities. The rise of telemedicine has highlighted how care delivery may change moving forward, making this period an ideal moment to recommit to ensure that quality care is available and accessible to all patients regardless of background.

\section{Definitions: Equity, Equality, Intersectionality}

Before engaging in health equity-related work, it is necessary to have a clear understanding of what exactly health equity is; therefore, the paragraphs below will define this and related terms.

\section{Equity and Equality}

The terms "equity" and "equality" are often mistakenly used interchangeably, and while related, it is important to understand the nuances of their different meanings. Health equity is "...the absence of systematic disparities in health, or in the major social determinants of health, between social groups who have different levels of underlying social advantage/disadvantage."8 Put more simply, "health equity means that everyone has a fair and just opportunity to be as healthy as possible." 9 Equality means that everyone is given equal opportunities and resources. For example, children in the U.S. have universal access to health insurance through either publicly or privately funded plans, so theoretically the country should have achieved equality in children's health. That this is not the case attests to the complex ways in which structural issues within medicine and society macroscopically have created a healthcare system inaccessible to some depending on their circumstances or identities. This present reality emphasizes the importance 
of prioritizing health equity specifically in seeking to improve pediatric health in the U.S. Thus, health equity is essentially health equality that accounts for the structural differences among individuals and understands that increased effort and resources need to be channeled towards less privileged members of society to ensure they have the same access to healthcare and positive health outcomes as those with more power, wealth, and privilege.

\section{Access}

In conceptualizing healthcare accessibility, we must remember that for patients simply getting some degree of care is not the same as getting timely, quality, affordable care. When defining access for equity, quality improvement, and research agendas, physicians and researchers need to ensure that they are using a broad definition inclusive of the financial, communication, time, and transportation barriers faced by patients.

\section{Intersectionality}

Intersectionality, a theory rooted in Black feminist scholarship, posits that not all individuals who are disenfranchised on the basis of their identity or circumstances have the same experiences of inequity. Instead, having different or numerous marginalized identities can contribute to variable experiences of privilege and power shaped by the complex ways that these categories of identity intersect in influencing social capital. ${ }^{10}$ For example, an individual who identifies as Black and transgender and comes from a low-income background will have a different experience of health and human rights than someone who is Black, cisgender, and from a wealthy background, as the intersection of race, gender, and economics are different for these individuals and no single characteristic defines their experience in society. In healthcare, intersectionality provides an analytic lens for providers to better understand the nuance of patient experiences and not focus solely on addressing healthcare disparities through the frame of any single identity, approach, or intervention.

\section{Inequities and Their Impact}

There is a significant body of existing literature within pediatric orthopaedics attesting to the existence of patient health inequities and exploring their roots and manifestations in clinical practice. Though the disparities discussed in the below paragraphs reflect discrete categories of patient identities and demographics as commonly structured in existing research, future studies should strive to adopt a more intersectional approach that accounts for how patients' different identities and circumstances shape their experience of orthopaedic surgical care.

\section{Race and Ethnicity}

Race and ethnicity-based health disparities are ubiquitous in healthcare and well documented in the pediatric literature. ${ }^{11}$ Of particular relevance within a surgical field like orthopaedics is the documented inequity in patient pain management, with non-white children being prescribed fewer opioids in ambulatory and emergency settings and reporting higher pain scores after general surgery operations. ${ }^{12-14}$ Research has also demonstrated poorer access to braces for cerebral palsy among African American and Hispanic children, and poorer functional outcomes following inpatient rehabilitation among children with Native American backgrounds. ${ }^{15,16}$

\section{Gender and Sexual Orientation}

Children who identify as LGBTQIA or gendernonconforming face barriers in accessing healthcare due to social stigmas and a lack of familial financial support. ${ }^{17}$ Transgender and gender-nonconforming children especially struggle due to mistrust of physicians stemming from experiences of being misgendered. ${ }^{18-20}$

\section{Insurance Type}

Children with public health insurance face limited access to orthopaedic surgical care leading to documented delays in fracture diagnosis and fixation, orthotic treatment, and diagnosis and treatment of meniscal tears. ${ }^{21-25}$ 


\section{Parental Health Literacy}

Both parental and patient health literacy are key to ensuring understanding of and engagement with treatment. One root of inequitable orthopaedic care lies in disparate levels of patient comprehension of orthopaedic terminology and care instructions, which are lower in families with lower education levels, incomes, and levels of English proficiency. ${ }^{26,27}$ This disparity is exacerbated by the inaccessibility of the patient education resources available on major society websites, many of which are written in complex language that lies above the average patient's level of reading comprehension. ${ }^{28,29}$

\section{Language}

Patients in the United States have the legal right to access healthcare in their preferred language, yet patients with low or limited English proficiency (LEP) face variable access to certified medical interpreters in orthopaedic care settings. ${ }^{30,31}$ Utilizing non-certified interpreters contributes to LEP patients/families facing decreased access to telehealth appointments, lower comprehension of orthopaedic terminology and treatments, and lower interpersonal satisfaction with the care that they receive. ${ }^{26,27,32,33}$ The adult literature further demonstrates resultant disparities in quality of care and postoperative outcomes among non-English speaking patients. ${ }^{6}$

\section{Nutrition and Food Access}

Disparities in rates of obesity and food access are particularly relevant to orthopaedists given the role these factors play in pediatric musculoskeletal health and the prevention of future adult musculoskeletal disease. There are significantly higher rates of obesity among those in African American, Hispanic, lowincome, and unemployed households. ${ }^{34,35}$ Those populations are also more likely to be food insecure or live in food deserts or swamps where there are limited supermarkets or locations to buy healthy food options but a high density of fast food options, limiting their ability to engage in nutrition and weight-focused treatment interventions. ${ }^{36}$

\section{Disability}

Patients with disabilities face widespread barriers to accessing both basic and specialty healthcare, not least of which is the significant financial cost of obtaining functional equipment such as braces and assistive devices which may not covered by insurance. Many report high distrust of the medical system stemming from ableist attitudes among providers regarding goals of care and quality of life, as well as difficulties navigating inaccessible facilities. ${ }^{37}$

\section{Space and Place}

Further research should investigate how access to safe housing, outdoor spaces, and neighborhoods impacts orthopaedic care given that these factors represent significant inequities that can impede patients' ability to exercise for weight loss, recovery, conditioning, and physical therapy purposes. Achieving and maintaining a healthy weight is an important preventative measure for numerous pediatric orthopaedic conditions since obesity both increases fracture risk and the risk of management failure, and contributes to conditions like SCFE, angular limb deformities, and musculoskeletal pain. ${ }^{38-40}$ Engaging in such facets of treatment requires both space within the home and access to places to exercise with clean air and a safe environment. Children who are non-white or living in households with lower incomes or parental education are less likely to have access to walking paths, parks, or recreation centers. ${ }^{41}$ They are also more likely to face housing and environmental inequities stemming from historically discriminatory housing policies that impact the air quality and safety of their neighborhoods and contribute to higher rates of asthma. ${ }^{11,42}$ It is vital to acknowledge that personal safety and the ability to exist outside comfortably can be fraught experiences for individuals of color, especially in the context of ongoing killings of Black individuals in their homes and neighborhoods as well as increased community violence against Asian-Americans during the COVID19 pandemic. 


\section{Interpersonal Interventions}

In the day-to-day clinical practice of a pediatric orthopaedic surgeon, a key area of intervention for reducing health inequities is at the interpersonal level of patient interactions. While many anecdotal recommendations exist within the orthopaedic literature regarding suggested engagement in health-equity focused work, further research systematically evaluating specific equity-focused interventions in orthopaedic settings is needed.

\section{Improving Communication}

A central goal of interpersonal inequity-reduction in the clinical setting should be increasing the quality and availability of care across a variety of domains. Poor physician communication is associated with lower rates of patient adherence with treatment; therefore, clinicians should constantly strive to ensure that their patient interactions are clear, accessible, and understandable. ${ }^{43}$

\section{Language}

Ensuring language accessibility often necessitates utilizing certified medical interpreters for those who are non-English speaking or with limited English proficiency (LEP). Professional interpreter usage is associated with improved patient comprehension of diagnoses, treatment, and discharge instructions as well as increased comprehension during the informed consent process. ${ }^{44,45}$ Their utilization is also associated with shorter lengths of hospitalization and decreased rates of 30-day readmission, compared to LEP patients with whom no professional interpreters are used. ${ }^{46}$ Of the limited data on interpreter usage in orthopaedics, one study found that when making an appointment to see an orthopaedist $80 \%$ of Spanish-speaking patients were instructed to bring a family member or friend to serve as their interpreter. ${ }^{30}$ Such ad-hoc interpreters are associated with higher rates of interpretation errors with potential clinical consequences compared to professionals. ${ }^{47}$ Varied interpreter usage often stems not from a lack of physician desire for better patient-provider communication, but instead from logistical issues involving organizing an interpreter and their perceived financial cost. ${ }^{48}$

While organizing and funding interpretation services will vary logistically between clinical settings and interpreter modality, some hospitals have increased inpatient usage through quality-improvement projects involving the interpreter utilization process. Standardizing the steps for requesting face-to-face interpretation, ensuring telephone/video interpreter availability in every unit, and sending out reminders and uniform education on interpreter usage to healthcare providers have been shown to increase interpreter utilization. ${ }^{49,50}$

The goal of accessible language also extends to the vocabulary physicians use during patient communications. We should limit the use of medical terminology that may be confusing to patients and explain things in clear language, utilizing models and visual aids to better demonstrate anatomy and the nature of surgical interventions. ${ }^{28,29}$ Beyond accessibility of content, language should also be inclusive of patients of all cultures and identities, meaning that physicians should use patients' preferred pronouns and correctly pronounced names in order to set a respectful and welcoming tone for the encounter.

In addition to spoken language, written educational materials should be clear and accessible to patients. AAOS website materials have been assessed as above the average patient's reading level, indicating that clinicians should review commonly used patient education materials for readability. ${ }^{28}$ Such materials are most effective when written at a 10- to 12-year old reading level; therefore, written content accessibility should be assessed via validated tools like the FleschKincaid grade, the New Dale-Chall Readability formula, or the Fry Readability graph, among many others. ${ }^{28,51}$ To increase accessibility to non-English and LEP patients, materials should also be translated into Spanish and other commonly spoken languages. One such existing resource is the POSNA OrthoKids website, an online source of basic information on common pediatric 
orthopaedic conditions that is available in both English and Spanish. ${ }^{52}$

\section{Scheduling, Telehealth, and Virtual Patient Portals}

It is also imperative to center inequity-reduction into scheduling and the integration of new technologies into the clinical workflow. Clinic hours often fall during normal work hours; therefore, limiting appointment scheduling services to these hours impedes access for working parents unable to take breaks, make calls, or wait on hold during the workday. Similarly, such families may struggle to take time off of work to take their children to appointments. Physicians should keep transportation and time barriers in mind when explaining treatment plans to patients, explicitly focusing on the time commitments of any proposed treatment timelines and minimizing unnecessary appointments or distances traveled. Clinics could also consider employing scheduling staff outside of 8:00 am to 4:30 pm work hours to increase the accessibility of scheduling and hold weekend or evening clinics to better accommodate working families.

Awareness of inequities is further necessary when integrating new technologies into the scheduling process or clinical workflow. Telehealth usage has exponentially increased during the COVID-19 pandemic, introducing new disparities in appointment access with Hispanic/ Latinx, Asian, non-English speaking patients, and patients insured through Medicaid having lower access to telehealth orthopaedic appointments than other patients. ${ }^{33,53}$ A similar effect exists with electronic patient portal usage within orthopaedic settings. While such portals decrease no-show rates and increase patient satisfaction with their care, they have had lower utilization among patients with lower levels of education, those who are non-English speaking, and those who are African American or Hispanic. ${ }^{54}$ Disparities in internet access threaten to worsen differences in portal accessibility, as those with advanced age or lower levels of education or income tend to have lower rates of internet access. ${ }^{55}$

Expanding health technologies during and beyond the pandemic necessitates providers maintain awareness that such actions threaten to exacerbate health disparities within already disadvantaged populations. Combating such paradoxical consequences requires prioritizing dissemination of telehealth options equally among patients regardless of background. To ensure that all patients receive quality care via telehealth, clinics may need to incorporate language interpretation services into their telemedicine platforms and have appointment options via telephone or video for those without access to an internet-enabled camera. ${ }^{33}$ Similarly, increasing the accessibility of electronic patient portals requires outreach and education encouraging enrollment, and translation of materials on patient portal usage in Spanish or other languages as appropriate for the patient population. ${ }^{54}$

\section{Eliminating Bias}

In addition to increasing the accessibility of orthopaedic care, clinicians should maintain awareness of, and actively work against, potential biases reflected in their language when charting. While it is easy to label a patient "non-compliant" with treatment if they miss physical therapy appointments or skip their assigned exercises, it is crucial to consider possible structural barriers to compliance impacting patients' lives and health. Circumstances often beyond a patient's control like transportation, space/housing constraints, and parental work schedules greatly contribute to their ability to engage in treatment. Labels regarding compliance frequently mask implicit racial biases, with past research using the race attitude Implicit Association Test (IAT) demonstrating that Internal and Emergency Medicine physicians showed implicit preferences for White patients compared to African American ones, with the latter being characterized as "less cooperative." 56 Within pediatricians specifically, research using the IAT has similarly demonstrated an implicit association between European Americans and the idea of a "compliant patient" compared to African Americans. ${ }^{57}$

To actively combat such implicit racial biases in the pediatric orthopaedic setting, when issues of compliance with treatment arise, clinicians should explicitly ask 
patients about challenges that impact their engagement in treatment and then work together with families to try to find resolutions. Such conversations should be initiated in a non-judgmental manner and emphasize that the inability to adhere with treatment is not always the fault of any one individual but often a result of socioeconomic conditions that are shaped by a families' identities and circumstances. It is reductive to simply assign a patient a normative label relating to compliance; therefore, such social determinants of health and their impact on treatment should be noted in the patient chart for better nuance and clarity regarding their background. As more EMRs incorporate social determinants of health tabs into their interfaces, orthopaedic surgeons can themselves input such details and search in that area of the chart to better understand their patients' backgrounds and potential resource challenges.

\section{Conclusion}

Providing the highest quality and most equitable care to all patients requires that orthopaedic surgeons adopt a broad, socially conscious approach to patient health and be cognizant that health does not exist within the confines of the exam room or surgical drapes but is instead inextricably shaped by a patient's identities, background, and social circumstances. By centering this perspective in patient care and integrating equity-focused changes within clinical settings, surgeons improve both their interpersonal interactions with marginalized patients and the quality of their care and subsequent health outcomes. Beyond interpersonal interactions, engaging in equity-focused work enables clinicians to better understand the unique health challenges faced by their patients. Such knowledge better equips orthopaedic surgeons to undertake both local and national advocacy work with the more macroscopic goal of reducing inequities by changing the policies that govern patients' lives and access to healthcare. This work is not a onetime occurrence but instead an ongoing, iterative process of interrogating one's approach to patient care to be best prepared to provide patients with the effective, highquality, and equitable care that every child deserves.

\section{References}

1. Cannada LK, O’Connor MI. Equity360: gender, race, and ethnicityharassment in orthopaedics and \#SpeakUpOrtho. Clin Orthop Relat Res. 2021;479(8)1674-1676. doi: https://doi.org/10.1097/ CORR.0000000000001884.

2. Gerull KM, Salles A, Porter SE, et al. Strategies for recruiting and retaining women and minorities in orthopaedics. J Bone Joint Surg Am. 2021;103(24):e98. doi: https://doi.org/10.2106/JBJS.21.00016.

3. McDonald TC, Drake LC, Replogle WH, et al. Barriers to increasing diversity in orthopaedics: the residency program perspective. JBJS Open Access. 2020;5(2):e0007. doi: https://doi.org/10.2106/JBJS. OA.20.00007.

4. Amen TB, Varady NH, Rajaee S, et al. Persistent racial disparities in utilization rates and perioperative metrics in total joint arthroplasty in the U.S.: a comprehensive analysis of trends from 2006 to 2015. JBJS. 2020;102(9).

5. Ali I, Vattigunta S, Jang JM, et al. Racial disparities are present in the timing of radiographic assessment and surgical treatment of hip fractures. Clin Orthop Relat Res. 2020;478(3):455-461.

6. Dowsey MM, Broadhead ML, Stoney JD, et al. Outcomes of total knee arthroplasty in english-versus non-english-speaking patients. J Orthop Surg. 2009;17(3):305-309. doi: https://doi.org/10.1177/230949900901700312.

7. Best MJ, Aziz KT, McFarland EG, et al. Worsening racial disparities in patients undergoing anatomic and reverse total shoulder arthroplasty in the United States. J Shoulder Elb Surg. 2021;30(8):1844-1850. doi: https://doi.org/10.1016/j.jse.2020.10.023.

8. Braveman P, Gruskin S. Defining equity in health. J Epidemiol Commun Health. 2003;57:254-258.

9. Braveman P, Arkin E, Orleans T, et al. What Is Health Equity? And What Difference Does a Definition Make? Princeton, NJ: Robert Wood Johnson Foundation; 2017.

10. Gkiouleka A, Huijts T, Beckfield J. Understanding the micro and macro politics of health: inequalities, intersectionality \& institutions - A research agenda. Soc Sci Med. 2018;200:92-98. doi: https://doi.org/10.1016/ j.socscimed.2018.01.025.

11. Fanta M, Ladzekpo D, Unaka N. Racism and pediatric health outcomes. Curr Probl Pediatr Adolesc Health Care. 2021;51(10):101087. doi: https://doi.org/10.1016/j.cppeds.2021.101087.

12. McCarthy J, Kelley J, Crawford A, et al. Racial inequalities in the delivery of pain medication in pediatric orthopaedics and a call to action. JPOSNA®. 2021;3(3). https://www.jposna.org/ojs/index.php/jposna/ article/view/304.

13. Drendel AL, Brousseau DC, Casper TC, et al. Opioid prescription patterns at emergency department discharge for children with Fractures. Pain Med. 2020;21(9):1947-1954.

14. Goyal MK, Johnson TJ, Chamberlain JM, et al. Racial and ethnic differences in emergency department pain management of children with fractures. Pediatrics. 2020;145(5):e20193370. doi: https://doi. org/10.1542/peds.2019-3370.

15. Rambo A, Rhodes L, Lomax J, et al. Factors that influence acquisition of lower extremity braces in the pediatric orthopaedic population. $J$ Pediatr Orthop. 2022;42(1). https://journals.lww.com/pedorthopaedics/ Fulltext/2022/01000/Factors_that_Influence_Acquisition_of_Lower.24. aspx.

16. Fuentes MM, Bjornson K, Christensen A, et al. Disparities in functional outcomes during inpatient rehabilitation between american indian/ alaska native and white children. $J$ Health Care Poor Underserved. 2016;27(3):1080-1096. doi: https://doi.org/10.1353/hpu.2016.0143.

17. Wagaman MA. Understanding service experiences of LGBTQ young people through an intersectional lens. Journal of Gay and Lesbian Social Services. 2014;26(1):111-145. doi: https://doi.org/10.1080/10538720. 2013.866867. 
18. Hoffman ND, Freeman K, Swann S. Healthcare preferences of lesbian, gay, bisexual, transgender and questioning youth. $J$ Adolesc Health. 2009;45(3):222-229. doi: https://doi.org/10.1016/j. jadohealth.2009.01.009.

19. Rider GN, McMorris BJ, Gower AL, et al. Health and care utilization of transgender and gender nonconforming youth: a population-based study. Pediatrics. 2018;141(3):e20171683. doi: https://doi.org/10.1542/ peds.2017-1683.

20. Lykens JE, LeBlanc AJ, Bockting WO. Healthcare experiences among young adults who identify as genderqueer or nonbinary. LGBT Health. 2018;5(3):191-196. doi: https://doi.org/10.1089/lgbt.2017.0215.

21. Skaggs DL, Lehmann CL, Rice C, et al. Access to orthopaedic care for children with medicaid versus private insurance: results of a national survey. J Pediatr Orthop. 2006;26(3):400-404. doi: https://doi. org/10.1097/01.bpo.0000217715.87857.24.

22. Sabatini CS, Skaggs KF, Kay RM, et al. Orthopedic surgeons are less likely to see children now for fracture care compared with 10 years ago. J Pediatr. 2012;160(3):505-507. doi: https://doi.org/10.1016/j. jpeds.2011.08.020.

23. Kitchen BT, Ornell SS, Shah KN, et al. Inequalities in pediatric fracture care timeline based on insurance type. J Am Acad Orthop Surg Glob Res Rev. 2020;4(8):e20.00111. doi: https://doi.org/10.5435/ JAAOSGlobal-D-20-00111.

24. Skaggs DL, Oda JE, Lerman L, et al. Insurance status and delay in orthotic treatment in children. J Pediatr Orthop. 2007;27(1):94-97. doi: https://doi.org/10.1097/01.bpo.0000242437.04059.41.

25. Olson M, Pandya N. Public insurance status negatively affects access to care in pediatric patients with meniscal injury. Orthop J Sports Med. 2021;9(1):2325967120979989. doi: https://doi. org/10.1177/2325967120979989.

26. Bagley CHM, Hunter AR, Bacarese-Hamilton IA. Patients' misunderstanding of common orthopaedic terminology: the need for clarity. Ann R Coll Surg Engl. 2011;93(5):401-404. doi: https://doi org/10.1308/003588411X580179.

27. Su L, Shaw K, Serpa RO, et al. Evaluation of general and musculoskeletal health literacy disparities in pediatric sports injury patient and guardian populations. J Pediatr Orthop. 2020;40(4):e237-e242.

28. Badarudeen S, Sabharwal S. assessing readability of patient education materials: current role in orthopaedics. Clin Orthop Relat Res. 2010;468:2572-2580. doi: https://doi.org/10.1007/s11999-010-1380-y.

29. Eltorai AEM, Sharma P, Wang J, et al. Most american academy of orthopaedic surgeons' online patient education material exceeds average patient reading level. Clin Orthop Relat Res. 2015;473:1181-1186. doi: https://doi.org/10.1007/s11999-014-4071-2.

30. Greene NE, Fuentes-Juárez BN, Sabatini CS. Access to orthopaedic care for Spanish-speaking patients in California. JBJS. 2019;101(18):e95.

31. $\mathrm{Ku} \mathrm{L}$, Flores $\mathrm{G}$. Pay now or pay later: providing interpreter services in health care. Health Aff. 2005;24(2):435-444. doi: https://doi.org/10.1377/ hlthaff.24.2.435

32. Baker DW, Hayes R, Fortier JP. Interpreter use and satisfaction with interpersonal aspects of care for spanish-speaking patients. Med Care. 1998;36(10):1461-1470

33. Xiong G, Greene NE, Lightsey HM, et al. Telemedicine use in orthopaedic surgery varies by race, ethnicity, primary language, and insurance status. Clin Orthop Relat Res. 2021;479(7):1417-1425. doi: https://doi.org/10.1097/CORR.0000000000001775.

34. Byrd AS, Toth AT, Stanford FC. Racial disparities in obesity treatment. Curr Obes Rep. 2018;7(2):130-38. doi: https://doi.org/10.1007/ s13679-018-0301-3.

35. Frederick CB, Snellman K, Putnam RD. Increasing socioeconomic disparities in adolescent obesity. Proc Natl Acad Sci USA. 2014;111(4):1338. doi: https://doi.org/10.1073/pnas.1321355110.

36. Larson NI, Story MT, Nelson MC. Neighborhood environments: disparities in access to healthy foods in the U.S. Am J Prevent
Med. 2009;36(1):74-81.e10. doi: https://doi.org/10.1016/j. amepre.2008.09.025

37. Doebrich A, Quirici M, Lunsford C. COVID-19 and the need for disability conscious medical education, training, and practice. J Pediat Rehabil Med. 2020;13(3):393-404. doi: https://doi.org/10.3233/ PRM-200763.

38. Nowicki P, Kemppainen J, Maskill L, et al. The role of obesity in pediatric orthopedics. J Am Acad Orthop Surg Glob Res Rev. 2019;3(5):e036. doi: https://doi.org/10.5435/JAAOSGlobal-D-19-00036.

39. Aversano MW, Moazzaz P, Scaduto AA, et al. Association between body mass index-for-age and slipped capital femoral epiphysis: the longterm risk for subsequent slip in patients followed until physeal closure. J Child Orthop. 2016;10(3):209-213. doi: https://doi.org/10.1007/ s11832-016-0731-y.

40. Taylor ED, Theim KR, Mirch MC, et al. Orthopedic complications of overweight in children and adolescents. Pediatrics. 2006;117(6): 2167-2174. doi: https://doi.org/10.1542/peds.2005-1832.

41. Singh GK, Siahpush M, Kogan MD. Neighborhood socioeconomic conditions, built environments, and childhood obesity. Health Affairs. 2010;29(3):503-512. doi: https://doi.org/10.1377/hlthaff.2009.0730.

42. Heard-Garris N, Boyd R, Kan K, et al. Structuring poverty: how racism shapes child poverty and child and adolescent health. Acad Pediat. 2021;21(8, Supplement):S108-S116. doi: https://doi.org/10.1016/j. acap.2021.05.026.

43. Zolnierek KB, Dimatteo MR. Physician communication and patient adherence to treatment: a meta-analysis. Med Care. 2009;47(8):826-834. doi: https://doi.org/10.1097/MLR.0b013e31819a5acc.

44. Baker DW, Parker RM, Williams MV, et al. Use and effectiveness of interpreters in an emergency department. JAMA. 1996;275(10): 783-788

45. Lee JS, Pérez-Stable EJ, Gregorich SE, et al. Increased access to professional interpreters in the hospital improves informed consent for patients with limited english proficiency. J Gen Intern Med. 2017;32(8):863-870. doi: https://doi.org/10.1007/s11606-0173983-4.

46. Lindholm M, Hargraves JL, Ferguson WJ, et al. Professional language interpretation and inpatient length of stay and readmission rates. $J$ Gen Intern Med. 2012;27:1294-1299. doi: https://doi.org/10.1007/ s11606-012-2041-5.

47. Flores G, Abreu M, Barone CP, et al. Errors of medical interpretation and their potential clinical consequences: a comparison of professional versus ad hoc versus no interpreters. Ann Emerg Med. 2012;60(5):545-553. doi: https://doi.org/10.1016/j.annemergmed.2012.01.025.

48. Jaeger FN, Pellaud N, Laville B, et al. Barriers to and solutions for addressing insufficient professional interpreter use in primary healthcare. BMC Health Serv Res. 2019;19(1):753. doi: https://doi.org/10.1186/ s12913-019-4628-6.

49. Rajbhandari P, Keith MF, Braidy R, et al. Interpreter use for limited english proficiency patients/families: a QI study. Hosp Pediatr. 2021;11(7):718-726. doi: https://doi.org/10.1542/hpeds.2020-003889.

50. Jacobs EA, Leos GS, Rathouz PJ, et al. Shared networks of interpreter services, at relatively low cost, can help providers serve patients with limited english skills. Health Affairs. 2011;30(10):1930-1938. doi: https:// doi.org/10.1377/hlthaff.2011.0667.

51. Johnson R, Edwards R, Rivers A, et al. Evaluating literacy levels of patient education materials for a sickle cell transition group education programme. Health Education Journal. 2020;79(3):253-265. doi: https:// doi.org/10.1177/0017896919876668.

52. OrthoKids. Pediatric Orthopaedic Society of North America. orthokids. org. Accessed January 3, 2022

53. Puzzitiello RN, Moverman MA, Pagani NR, et al. Public perceptions and disparities in access to telehealth orthopaedic services in the COVID-19 era. J Natl Med Assoc. 2021;113(4):405-413. doi: https://doi.org/10.1016/j. jnma.2021.02.007. 
54. Varady NH, d'Amonville S, Chen AF. Electronic patient portal use in orthopaedic surgery is associated with disparities, improved satisfaction, and lower no-show rates. JBJS. 2020;102(15). https://journals.lww.com/ jbjsjournal/Fulltext/2020/08050/Electronic_Patient_Portal_Use_in_ Orthopaedic.11.aspx.

55. Walsh KP, Saqib R, Jessie G. Disparities in internet use among orthopedic outpatients. Orthopedics. 2014;37(2):e133-e140. doi: https://doi. org/10.3928/01477447-20140124-14.
56. Green AR, Carney DR, Pallin DJ, et al. Implicit bias among physicians and its prediction of thrombolysis decisions for black and white patients. $J$ Gen Intern Med. 2007;22:1231-1238.

57. Sabin JA, Rivara FP, Greenwald AG. Physician implicit attitudes and stereotypes about race and quality of medical care. Med Care. 2008;46(7):678-685. doi: https://doi.org/10.1097/ MLR.0b013e3181653d58. 